\title{
Mackey continuity of convex functions on dual Banach spaces: a review
}

\author{
A.J. WroBeL * \\ 15082 East County Road 600N, Charleston, Illinois, 61920-8026, United States \\ a.wrobel@alumni.lse.ac.uk \\ https://www.researchgate.net/profile/Andrew_Wrobel3/research
}

Received December 13, 2019

Accepted September 13, 2020

Presented by David Yost

\begin{abstract}
A convex (or concave) real-valued function, $f$, on a dual Banach space $P^{*}$ is continuous for the Mackey topology $\mathrm{m}\left(P^{*}, P\right)$ if (and only if) it is Mackey continuous on bounded subsets of $P^{*}$. Equivalence of Mackey continuity to sequential Mackey continuity follows when $P$ is strongly weakly compactly generated, e.g., when $P=L^{1}(T)$, where $T$ is a set that carries a sigma-finite measure $\sigma$. This result of Delbaen, Orihuela and Owari extends their earlier work on the case that $P^{*}$ is either $L^{\infty}(T)$ or a dual Orlicz space. An earlier result of this kind is recalled also: it derives Mackey continuity from bounded Mackey continuity for a nondecreasing concave function, $F$, that is defined and finite only on the nonnegative cone $L_{+}^{\infty}$. Applied to a linear $f$, the Delbaen-OrihuelaOwari result shows that the convex bounded Mackey topology is identical to the Mackey topology, i.e., $\operatorname{cbm}\left(P^{*}, P\right)=\mathrm{m}\left(P^{*}, P\right)$; here, this is shown to follow also from Grothendieck's Completeness Theorem. As for the bounded Mackey topology, bm $\left(P^{*}, P\right)$, it is conjectured here not to be a vector topology, or equivalently to be strictly stronger than $\mathrm{m}\left(P^{*}, P\right)$, except when $P$ is reflexive.
\end{abstract}

Key words: Dual Banach space, convex bounded Mackey topology, convergence in measure, economic equilibrium.

AMS Subject Class. (2010): Primary 46B99, 46E30, 52A41; Secondary 46A70.

\section{INTRODUCTION}

Nonmetric topologies on the norm-dual, $P^{*}$, of a real Banach space $(P)$ can become much more manageable when restricted to bounded sets. For example, given a convex subset of $P^{*}$, or a real-valued concave function on $P^{*}$, the bounded weak* topology, $\mathrm{bw}^{*}:=\mathrm{bw}\left(P^{*}, P\right)$, can serve to show that the set in question is weakly* closed, or that the function is weakly* upper semicontinuous.

In economic theory, such uses of the Krein-Smulian Theorem are made in [7, Proposition 1.1, Theorem 4.4 and Theorem 4.7], [12, Proposition 1 and Example 5], [14, Lemma 4.1] and [15, Section 6.2]. In applications of economic

${ }^{*}$ Formerly of the London School of Economics.

ISSN: 0213-8743 (print), 2605-5686 (online)

(C) The author(s) - Released under a Creative Commons Attribution License (CC BY-NC 3.0) 
equilibrium models, this can be an indispensable tool for verifying that the production sets that describe the technologies are weakly* closed, and that the profit and cost functions are weakly* semicontinuous (which is needed for equilibria to exist, and for the dual pairs of programmes to have no duality gaps): see [11, Lemma 6.1] and [15, Lemmas 6.2.3-6.2.5].1

When $P$ is $L^{1}(T, \sigma)$, the space of integrable real-valued functions on a set $T$ that carries a sigma-finite measure $\sigma$-and so $P^{*}$ is the space of essentially bounded functions $L^{\infty}(T)$-another useful "bounded" topology on $L^{\infty}$ is the bounded Mackey topology, bm $\left(L^{\infty}, L^{1}\right)$. This is because a convex (or concave) real-valued function, $f$, is continuous for the "plain" Mackey topology, $\mathrm{m}\left(L^{\infty}, L^{1}\right)$, if (and only if) it is bm $\left(L^{\infty}, L^{1}\right)$-continuous, i.e., $\mathrm{m}\left(L^{\infty}, L^{1}\right)$ continuous on bounded sets - or, equivalently, if (and only if) $f$ is continuous along bounded sequences (in $L^{\infty}$ ) that converge in measure (on subsets of $T$ of finite measure). Thus the reduction to bounded sets provides direct access to the methods of integral calculus, which can greatly simplify verification of Mackey continuity [12, Example 5]. And, in economic equilibrium analysis, Mackey continuity of a concave utility or production function is essential for representing the price system by a density, as is done in [3] and [13]. In addition, the use of convergence in measure furnishes economic interpretations of Mackey continuity [12, Section 4 and Section 5].

When the convex function $f$ is defined and finite on the whole space $L^{\infty}$, the equivalence of $\mathrm{m}\left(L^{\infty}, L^{1}\right)$-continuity to $\mathrm{bm}\left(L^{\infty}, L^{1}\right)$-continuity is a result of Delbaen and Owari [7, Proposition 1.2], which is quoted here as Theorem 2 they also extend it to the case of a dual Orlicz space instead of $L^{\infty}$ [7. Theorem 4.5] and apply it in the mathematics of finance [7, Theorem 4.8]. Their ingenious argument shows first that the sublevel sets of the conjugate function, $f^{\#}$, are uniformly integrable and hence weakly compact (in $L^{1}$ ); it then follows that $f$ is Mackey continuous (on $L^{\infty}$ ) by the Moreau-Rockafellar Theorem (on the conjugacy between continuity and inf-compactness).

An earlier result that derives Mackey continuity from its bounded version applies to a nondecreasing concave function, $F$, that is defined (and finite) only on the nonnegative cone $L_{+}^{\infty}$. Quoted here as Theorem 1, it requires a different method - one which relies on Mackey continuity of the lattice operations in $L^{\infty}$ as well as on the monotonicity of $F$ [12, Proposition 1 and Proposition 3 with Example 2 and Example 4]. (This case is different because $F$ need not have a finite concave extension to the whole space $L^{\infty}$, and the 11.

${ }^{1} \mathrm{~A}$ similar use of $\mathrm{bw}^{*}$ is made in Part I of the work on energy storage whose Part II is 
Mackey interior of $L_{+}^{\infty}$ is empty - except when $L^{\infty}(T)$ is finite-dimensional, i.e., when $T$ consists of a finite number of atoms of $\sigma$.)

For a finite-valued convex (or concave) $f$ defined on the whole space, the equivalence of Mackey continuity to bounded Mackey continuity extends to the case of a general dual Banach space, $P^{*}$, as the domain of $f$ : see Delbaen and Orihuela [6, Theorem 8]. (Equivalence to sequential Mackey continuity follows when $P$ is strongly weakly compactly generated [6, Corollary 11].) A fortiori, those linear functionals (on $P^{*}$ ) that are continuous for the bounded Mackey topology, $\mathrm{bm}^{*}:=\mathrm{bm}\left(P^{*}, P\right)$, are actually continuous for $\mathrm{m}^{*}:=\mathrm{m}\left(P^{*}, P\right)$, i.e., belong to $P$. It follows that the convex bounded Mackey topology, $\operatorname{cbm}\left(P^{*}, P\right)$, is identical to the "plain" Mackey topology 2: $\mathrm{cbm}^{*}=\mathrm{m}^{*}$ for every $P .{ }^{3}$ Implicit in [12, Proposition 1], this result is derived here more simply from Grothendieck's Completeness Theorem (Proof of Proposition 1). It does not follow that $\mathrm{bm}^{*}$ equals $\mathrm{m}^{*}$ because $\mathrm{bm}^{*}$ is not known to be a vector topology and, indeed, it is conjectured not to be one, unless $P$ is reflexive (Conjecture 1).

There are, then, two different methods of "upgrading" the bounded Mackey continuity to full, unqualified Mackey continuity, each with its own limitations and its own area of applicability:

- The method of [12] applies to a nondecreasing concave function that is finite only on the cone $L_{+}^{\infty}$ (or, more generally, on the nonnegative cone $P_{+}^{*}$ of a dual Banach lattice $P^{*}$ on which $\mathrm{m}\left(P^{*}, P\right)$ is locally solid, i.e., is a vector-lattice topology).

- The method of [6] and [7] applies to an everywhere-finite convex (or concave) function on a dual Banach space $P^{*}$; it is based on the FenchelLegendre conjugacy (and on using convergence in measure when $P^{*}$ is $L^{\infty}$ or a dual Orlicz space).

Finally, the case of a dual Banach lattice different from $L^{\infty}$, in which the norm-bounded sets differ from the order-bounded ones, is addressed briefly in the Remarks at the end of Section 2, these include an outline of the DelbaenOwari analysis for dual Orlicz spaces in [7].

\footnotetext{
${ }^{2}$ For $P=L^{1}$ only, that $\operatorname{cbm}\left(L^{\infty}, L^{1}\right)=\mathrm{m}\left(L^{\infty}, L^{1}\right)$ has been shown earlier by methods specific to this space, in [5, III.1.6 and III.1.9] and in [16, Theorem 5].

${ }^{3}$ It also follows that $\mathrm{bm}^{*}$-continuity upgrades to $\mathrm{m}^{*}$-continuity not only for linear functionals but also for general linear maps, i.e., every $\mathrm{bm}^{*}$-continuous linear map of $P^{*}$, into any topological vector space, is $\mathrm{m}^{*}$-continuous (on $P^{*}$ ). This is because, for a linear map of a space with topologies of the forms $\mathrm{b} \mathcal{T}$ and $\operatorname{cb} \mathcal{T}$, its $\mathrm{b} \mathcal{T}$-continuity implies $\operatorname{cb} \mathcal{T}$-continuity [5. I.1.7], and because $\mathrm{cbm}^{*}=\mathrm{m}^{*}$.
} 


\section{MACKEY CONTINUITY DERIVED FROM BOUNDED CONTINUITY}

Quoted below are the continuity-upgrade results for finite nondecreasing concave functions on $L_{+}^{\infty}$ [12] and for finite convex functions on $L^{\infty}$ [7] or on any Banach dual space $P^{*}[6]$.

Terminology (weak topology and Mackey topology, SWCG space):

- Let $P^{*}$ be the norm-dual of a real Banach space $P$. The weakest and the strongest of those locally convex topologies on $P^{*}$ which yield $P$ as the continuous dual are denoted by $\mathrm{w}\left(P^{*}, P\right)$ and $\mathrm{m}\left(P^{*}, P\right)$. Known as the weak and the Mackey topologies, on $P^{*}$ for its pairing with $P$, the two can be called the weak* and the Mackey topologies (since $\mathrm{m}\left(P^{*}, P^{* *}\right)$, the Mackey topology on $P^{*}$ for its pairing with its norm-dual $P^{* *}$, is identical to the norm topology of $\left.P^{*}\right)$. With $\mathrm{w}\left(P, P^{*}\right)$ denoting the weak topology of $P, \mathrm{~m}\left(P^{*}, P\right)$ can be described as the topology of uniform convergence on all $\mathrm{w}\left(P, P^{*}\right)$-compact, convex and balanced (a.k.a. circled) subsets of $P$ [17, IV.3.2: Corollary 1] $]^{4}$

- A real Banach space $P$-whose norm-dual is $P^{*}$ and whose unit ball (centered at 0 ) is $B$-is called strongly weakly compactly generated (SWCG) if it contains a w $\left(P, P^{*}\right)$-compact set $G$ such that, for every $\mathrm{w}\left(P, P^{*}\right)$-compact set $C \subset P$ and every scalar $\epsilon>0$, there exists an $n \in \mathbb{N}$ with $C \subseteq n G+\epsilon B$. (When such a $G$ exists, it can be chosen to be convex and balanced, by Krein's Theorem [17, IV.11.4].) See [18] for properties and examples of such spaces.

Theorem 1. (Horsley-Wrobel) Let $\sigma$ be a sigma-finite nonnegative measure on $T$. For a nondecreasing concave function $F: L_{+}^{\infty}(T, \sigma) \rightarrow \mathbb{R}$ (defined and finite only on the nonnegative cone $L_{+}^{\infty}$ ), the following conditions are equivalent to one another:

(1) $F$ is $\mathrm{m}\left(L^{\infty}, L^{1}\right)$-continuous (on $\left.L_{+}^{\infty}\right)$;

(2) $F$ is $\mathrm{m}\left(L^{\infty}, L^{1}\right)$-continuous on bounded subsets (of $\left.L_{+}^{\infty}\right)$;

(3) $F$ is sequentially $\mathrm{m}\left(L^{\infty}, L^{1}\right)$-continuous (on $\left.L_{+}^{\infty}\right)$;

(4) on bounded subsets (of $L_{+}^{\infty}$ ), $F$ is continuous for $\mathcal{T}_{\sigma}$, the topology of convergence in the measure $\sigma$ on subsets of $T$ of finite measure.

\footnotetext{
${ }^{4}$ Here "convex" can actually be omitted because $P$ is a Banach space and one can apply Krein's Theorem [17, IV.11.4]. That "circled" can be omitted is obvious [17, I.5.2].
} 
Proof. See [12, Proofs of Proposition 1 and Proposition 3, with Example 2 and Example 4].

When the cone $L_{+}^{\infty}$ is replaced by the whole space $L^{\infty}$, the same holds without the monotonicity assumption. Despite their formal similarity, the two cases are very different in their methods of proof, which overlap only in their standard uses of: (i) the Krein-Smulian Theorem (for the "easy" semicontinuity part), and (ii) the equality, on bounded sets, of $\mathrm{m}\left(L^{\infty}(\sigma), L^{1}(\sigma)\right)$ to $\mathcal{T}_{\sigma}$ (the topology of convergence in measure).

Theorem 2. (Delbaen-OWARI) Let $\sigma$ be a sigma-finite nonnegative measure on $T$. For a convex (or concave) function $f: L^{\infty}(T, \sigma) \rightarrow \mathbb{R}$ that is finite everywhere (on the whole space $L^{\infty}$ ), the following conditions are equivalent to one another:

(1) $f$ is $\mathrm{m}\left(L^{\infty}, L^{1}\right)$-continuous (on $\left.L^{\infty}\right)$;

(2) $f$ is $\mathrm{m}\left(L^{\infty}, L^{1}\right)$-continuous on bounded subsets (of $\left.L^{\infty}\right)$;

(3) $f$ is sequentially $\mathrm{m}\left(L^{\infty}, L^{1}\right)$-continuous (on $\left.L^{\infty}\right)$;

(4) on bounded subsets (of $L^{\infty}$ ), $f$ is continuous for $\mathcal{T}_{\sigma}$, the topology of convergence in the measure $\sigma$ on subsets of $T$ of finite measure.

Proof. The argument of [7, Proof of Proposition 1.2], where $\sigma$ is assumed to be finite, is sufficient when $\sigma$ is also nonatomic. It can be extended to the case an arbitrary sigma-finite $\sigma$ by using two standard techniques: (i) embedding a finite measure with atoms in a nonatomic one, and (ii)replacing a sigma-finite measure by an equivalent finite one (i.e., one with a strictly positive and integrable density).

Conditions (1) and (2) of Theorem 2 remain equivalent to each other when $L^{\infty}$ is replaced by any dual Banach space $P^{*}$ (with "bounded" taken to mean "norm-bounded"). Condition (3) is equivalent to the first two if $P$ is SWCG.

Theorem 3. (Delbaen-Orinuela-Owari) Let $P$ be a real Banach space and $P^{*}$ its norm-dual. For a convex (or concave) function $f: P^{*} \rightarrow \mathbb{R}$ that is finite everywhere (on the whole space $P$ ), the following two conditions are equivalent to each other:

(1) $f$ is $\mathrm{m}\left(P^{*}, P\right)$-continuous (on $\left.P^{*}\right)$; 
(2) $f$ is $\mathrm{m}\left(P^{*}, P\right)$-continuous on norm-bounded subsets (of $\left.P^{*}\right)$.

When $P$ is strongly weakly compactly generated, the above Condition (2) is equivalent (whether $f$ is convex or not) to the condition ${ }^{5}$ :

(3) $f$ is sequentially $\mathrm{m}\left(P^{*}, P\right)$-continuous $\left(\right.$ on $\left.P^{*}\right)$.

Proof. See [6, Theorem 8 and Corollary 11]. K. Owari has given a simpler proof that Condition (2) implies Condition (1): although his argument is essentially the same as that in [6], it does not involve the approximate subdifferential ${ }^{6}$ Also, the assumption in [6, Theorem 8 and Corollary 11] that $f$ is a conjugate function (or, equivalently, that it is weakly* lower semicontinuous on $P^{*}$ ) is superfluous: it follows from the bounded Mackey continuity (and the convexity) of $f$ by the Krein-Smulian Theorem (as is noted in 7, Proposition 1.1] and in [12, Proposition 1]).

Remarks (on the case of a dual Banach lattice $P^{*} \neq L^{\infty}$ ):

- In such a Banach lattice, order-bounded sets are norm-bounded, but not vice versa. Four more questions arise, then, about $\mathrm{m}\left(P^{*}, P\right)$-continuity of a finite convex function $f$ on $P^{*}$ : (a) Does sequential continuity on order-intervals imply either (i) continuity on order-intervals, or (ii) sequential continuity (on balls and hence on all of $P^{*}$ by the BanachSteinhaus Theorem)? (b) Does either of the last two conditions imply continuity on $P^{*}$ ? For dual Orlicz spaces, all four conditions are equivalent by a result of Delbaen and Owari [7, Theorem 4.5], whose analysis can be outlined as follows.

- On order-intervals, sequential continuity implies continuity (i.e., "yes" to Part (i) of (a)) whenever $\mathrm{m}\left(P^{*}, P\right)$ is metrizable on order-intervals. And $\mathrm{m}\left(P^{*}, P\right)$ is so not only when $P$ is SWCG, but also when $P$ is an Orlicz space $L^{\Phi}(T, \sigma)$-where $\sigma$ is a finite nonnegative measure on $T$ and $\Phi: \mathbb{R}_{+} \rightarrow \mathbb{R}_{+}$is a super-coercive (a.k.a. strict) Young function that meets the $\Delta_{2}$-condition, and so $\left(L^{\Phi}\right)^{*}=L^{\Phi^{\#}}$, where $\Phi^{\#}$ is the convex conjugate of $\Phi$ [7, pp. 1052-1053]. On each order-interval of such a space, $\mathrm{m}\left(L^{\Phi^{\#}}, L^{\Phi}\right)$ is equal to $\mathcal{T}_{\sigma}$, the globally metrizable topology of convergence in the measure $\sigma$ [7, (2.1)].

\footnotetext{
${ }^{5}$ The corresponding part of Theorem 2 is a special case of this, since $L^{1}(T, \sigma)$ is SWCG when $\sigma$ is sigma-finite [18, 2.3].

${ }^{6}$ Personal communication.
} 


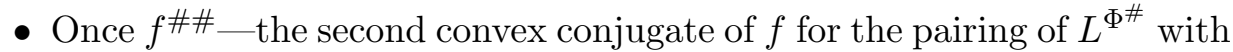
$L^{\Phi}$ — has been shown to equal $f$, that sequential $\mathrm{m}\left(L^{\Phi^{\#}}, L^{\Phi}\right)$-continuity on order-intervals implies unqualified $\mathrm{m}\left(L^{\Phi^{\#}}, L^{\Phi}\right)$-continuity is shown by using $\mathcal{T}_{\sigma}$ in essentially the same way as in the case of $P^{*}=L^{\infty}$ : compare the proofs of [7, Proposition 1.2 and Theorem 4.5].

- So, to complete the proof that all four conditions are equivalent (for $\left.P=L^{\Phi}\right)$, it remains to establish that indeed $f^{\# \#}=f$ when $f$ is $\mathrm{m}\left(L^{\Phi^{\#}}, L^{\Phi}\right)$-lower semicontinuous (l.s.c.) on order-intervals. This is done, in [7, Theorem 4.1 and Theorem 4.4], by using a new variant of Komlós's Theorem [7, Theorem 3.6 and Corollary 3.10] — which, crucially, from a merely norm-bounded sequence (in $L^{\Phi^{\#}}$ ) produces an order-bounded and $\sigma$-almost everywhere convergent sequence of forward convex combinations. Of interest in itself, in the present context the Delbaen-Owari variant of Komlós's Theorem bridges the gap between order- and norm-boundedness 7

- The question of whether, on $P^{*}$, sequential continuity implies continuity (i.e., Part (ii) of (b)) does not involve the order structure, and by Theorem 3 the answer is "yes" when $P$ is SWCG. The outlined analysis of [7, which does use the order, adds the "yes" answer for $P=L^{\Phi}$ whether it is SWCG or not, which in general seems to be an unanswered question 8

- Continuity on order-intervals (rather than balls) can be of use in verifying "full" continuity also when $P$ is a reflexive Banach lattice (e.g., the Lebesgue space $L^{\varrho}$ with $\left.1<\varrho<+\infty\right)$, in which case $\mathrm{m}\left(P^{*}, P\right)$ is the norm topology.

- With a Banach lattice $P$ other than $L^{\Phi}$, any extension of this analy-

\footnotetext{
${ }^{7}$ In detail: since $f$ is convex and $\mathrm{m}\left(L^{\Phi^{\#}}, L^{\Phi}\right)$-l.s.c. on order-intervals, it follows from 7. Corollary 3.10] — by using ordinary sequences (rather than uncountable nets) — that $f$ is $\mathcal{T}_{\sigma^{-}}$ l.s.c. on norm -bounded sets. $A$ fortiori, $f$ is $\mathrm{m}\left(L^{\Phi^{\#}}, L^{\Phi}\right)$-l.s.c. on such sets and so, being convex, it is w $\left(L^{\Phi^{\#}}, L^{\Phi}\right)$-l.s.c. on norm-bounded sets. By the Krein-Smulian Theorem [17, IV.6.4], this means that $f$ is actually $\mathrm{w}\left(L^{\Phi^{\#}}, L^{\Phi}\right)$-l.s.c. on all of $L^{\Phi^{\#}}$-i.e., that $f^{\# \#}=f$.

${ }^{8}$ The space $L^{\Phi}$ is known to be SWCG if $y \Phi^{\prime}(y) / \Phi(y) \rightarrow 1$ as $y \rightarrow+\infty$ or, equivalently, if $1=\varrho_{\Phi}:=\limsup _{y \rightarrow+\infty} y \Phi^{\prime}(y) / \Phi(y)$, where $\Phi^{\prime}$ is the one-sided (left or right) derivative of $\Phi$ : see [6, paragraph after (20)]. (That $\varrho_{\Phi}<+\infty$ is an equivalent form of the $\Delta_{2}$-condition [7. (1.1)].) But this criterion proves $L^{\Phi}$ to be SWCG only when its $\varrho_{\Phi}$ is the same as that of the SWCG space $L^{1}$.
} 
sis would have to start with a metric for $\mathrm{m}\left(P^{*}, P\right)$ on order-intervals. But its very existence seems to be an unanswered question (when $P$ is not SWCG). This is so even though there is a relevant metrizability criterion-for order-continuous locally solid topologies, a.k.a. Lebesgue topologies. Namely, a Lebesgue topology, $\mathcal{T}$, on a vector lattice $Y$ is metrizable on order-intervals if and only if $Y$ has the countable sup property [2, 4.26]. And a locally convex-solid topology, $\mathcal{T}$, on a vector lattice $Y$ is order-continuous if (and only if) the $\mathcal{T}$-dual of $Y$ is contained in the order-continuous dual, $Y_{\mathrm{n}}^{\sim}$, of $Y$ [2, 3.12]. This inclusion always holds for $Y=P^{*}$ and $\mathcal{T}=\mathrm{m}\left(P^{*}, P\right)$, i.e., $P \subseteq\left(P^{\sim}\right)_{\mathrm{n}}^{\sim}=\left(P^{*}\right)_{\mathrm{n}}^{\sim}$ by [1, p. 331, line 8 f.b.], since the order-dual $P^{\sim}$ is equal to the normdual $P^{*}$ [1, Theorem 9.11]. So the metrizability criterion applies to $\mathrm{m}\left(P^{*}, P\right)$ when (and only when) $\mathrm{m}\left(P^{*}, P\right)$ is locally solid (i.e., makes $P^{*}$ a topological vector lattice): on this condition, $\mathrm{m}\left(P^{*}, P\right)$ is metrizable on order-intervals if and only if $P^{*}$ has the countable sup property (or, equivalently, is of countable type). But, apart from $L^{\infty}$ (and dual Orlicz spaces), few, if any, examples seem to be known of (nonreflexive) dual Banach lattices whose Mackey topologies are locally solid.

\section{The Bounded And COnVEx Bounded Mackey topologies ON A DUAL BANACH SPACE}

Mackey continuity on norm-bounded subsets of the norm-dual, $P^{*}$, of a real Banach space $P$ (which is Condition (2) of Theorem 3) can be restated as continuity for the bounded Mackey topology. This is a case of [8, Theorem 1(b)], and it holds for every map (of $P^{*}$ ) into any topological space: it does not depend on any monotonicity or convexity properties of the map. Denoted by $\mathrm{bm}\left(P^{*}, P\right)$ or $\mathrm{bm}^{*}$ for brevity, the bounded Mackey topology is the strongest topology that is equal to $\mathrm{m}\left(P^{*}, P\right)$ on every bounded subset (of $\left.P^{*}\right)$. The convex bounded Mackey topology, denoted by $\operatorname{cbm}\left(P^{*}, P\right)$ or $\mathrm{cbm}^{*}$ for brevity, is the strongest locally convex topology that is equal to $\mathrm{m}\left(P^{*}, P\right)$ on every bounded set. Remarkably, this is also the strongest vector topology that is equal to $\mathrm{m}\left(P^{*}, P\right)$ on every bounded set: this is a case of [19, 2.2.2], a result given also in [5, I.1.4 and I.1.5 (iii)]. Obviously bm* is at least as strong as cbm*, which in turn is at least as strong as $\mathrm{m}^{*}:=\mathrm{m}\left(P^{*}, P\right)$. The last two are actually one and the same. A corollary to Theorem 3 , this equality is next obtained more simply by applying Grothendieck's Completeness Theorem [17, IV.6.2], which is quoted for easy reference. 
TheOREM 4. (GROTHENDIECK) Let $\mathfrak{T}$ be a locally convex topology on a real vector space $E$. When additionally $\mathfrak{S}$ is a saturated family ${ }^{9}$ of $\mathfrak{T}$ bounded sets covering $E$, the $\mathfrak{T}$-dual of $E$ is complete under the $\mathfrak{S}$-topology (the topology of uniform convergence on every $S \in \mathfrak{S}$ ) if and only if every linear functional (on $E$ ) that is $\mathfrak{T}$-continuous on each $S \in \mathfrak{S}$ is actually $\mathfrak{T}$ continuous on the whole space $E$ (i.e., is in the $\mathfrak{T}$-dual of $E$ ).

Proposition 1. Let $P$ be a real Banach space, and $P^{*}$ its norm-dual. Then $\operatorname{cbm}\left(P^{*}, P\right)=\mathrm{m}\left(P^{*}, P\right)$.

Proof. Apply Theorem 4 to $P^{*}$ as $E$-with $\mathrm{m}^{*}$ as $\mathfrak{T}$ and the bounded subsets of $P^{*}$ as $\mathfrak{S}$, and hence with $P$ as the $\mathfrak{T}$-dual and the norm topology of $P$ as the $\mathfrak{S}$-topology - to conclude that a linear functional on $P^{*}$ is $\mathrm{m}^{*}$ continuous if it is so on bounded sets (i.e., if it is $\mathrm{bm}^{*}$-continuous). A fortiori, it is $\mathrm{m}^{*}$-continuous (i.e., is in $P$ ) if it is $\mathrm{cbm}^{*}$-continuous. In other words, cbm* yields the same dual space as $\mathrm{m}^{*}$ (viz., $P$ ). This proves that $\mathrm{cbm}^{*}=\mathrm{m}^{*}$ (since $\mathrm{cbm}^{*}$ is both locally convex and stronger than $\mathrm{m}^{*}$ ). 10

As for $\operatorname{bm}\left(P^{*}, P\right)$, it is semi-linear (i.e., both vector addition and scalar multiplication are separately continuous in either variable): this is a case of [4, Theorem 5], a result noted also in [8, p. 410]. It seems to be unknown whether $\mathrm{bm}^{*}$ is linear (i.e., a vector topology), but if it were, it would be identical to $\mathrm{cbm}^{*}=\mathrm{m}^{*}$. The Delbaen-Owari result, with its reliance on convexity, makes this implausible - as is put forward next.

Conjecture 1. For $P=L^{1}[0,1]$ at least, and possibly for every nonreflexive Banach space $P$, the topology $\operatorname{bm}\left(P^{*}, P\right)$ is strictly stronger than $\mathrm{m}\left(P^{*}, P\right)$-or, equivalently, $\mathrm{bm}\left(P^{*}, P\right)$ is not linear.

The conjecture, then, is based on what it takes to establish that a bm*continuous $\mathbb{R}$-valued function $f$, on a nonreflexive space $P^{*}$, is $\mathrm{m}^{*}$-continuous: Theorem 2 and Theorem 3 require $f$ to be convex, and it is hard to imagine

\footnotetext{
${ }^{9}$ A family, $\mathfrak{S}$, of subsets of a locally convex space is called saturated [17, p. 81] if: (i) all subsets of every member of $\mathfrak{S}$ belong to $\mathfrak{S}$, (ii) all scalar multiples of every member of $\mathfrak{S}$ belong to $\mathfrak{S}$, and (iii) for each finite $\mathfrak{F} \subset \mathfrak{S}$, the closed convex circled hull of the union of $\mathfrak{F}$ belongs to $\mathfrak{S}$.

${ }^{10}$ Alternatively, Cooper's special case of Grothendieck's Theorem [5, I.1.17 (ii)] can be applied - to $P^{*}$ as his $E$, with $\mathrm{m}^{*}$ as $\tau$ and the bounded subsets of $P^{*}$ as $\mathcal{B}$, and hence with $P^{* *}$ as $E_{\mathcal{B}}^{\prime}$ and $\mathrm{cbm}^{*}$ as his $\gamma=\gamma(\mathcal{B}, \tau)$ - to conclude that the $\mathrm{cbm}^{*}$-dual equals the $\mathrm{m}^{*}$-dual $\left(\right.$ so $\left.\mathrm{cbm}^{*}=\mathrm{m}^{*}\right)$.
} 
(even when $P^{*}=L^{\infty}[0,1]$ ) how the convexity assumption might be disposed of entirely — as would be necessary for $\mathrm{bm}^{*}$ to equal $\mathrm{m}^{*}$.

Comment on Bounded topologies: See [20] for a more detailed review of such topologies: in addition to $\mathrm{bw}^{*}$ and $\mathrm{bm}^{*}$, it includes the bounded weak topology and its convex variant (bw and cbw), which are studied in 9 , as well as the compact weak topology and its convex variant (kw and ckw), which are introduced in [10].

\section{ACKNOWLEDGEMENTS}

I am obliged to Keita Owari for telling me of Theorem 3 and for the reference [6]. I am also grateful to the referee for useful comments, and to the editor for his handling of the manuscript.

\section{REFERENCES}

[1] C.D. Aliprantis, K.C. Border, "Infinite Dimensional Analysis", Springer, Berlin-Heidelberg-New York, 2006. doi.org/10.1007/3-540-29587-9

[2] C.D. Aliprantis, O. Burkinshaw, "Locally Solid Riesz Spaces with Applications to Economics", American Mathematical Society, Providence, RI, 2003. doi.org/10.1090/surv/105

[3] T.F. BEWLEy, Existence of equilibria in economies with infinitely many commodities, J. Econom. Theory 4(3) (1972), 514-540. doi.org/10.1016/0022-0531(72)90136-6

[4] H.S. Collins, Completeness and compactness in linear topological spaces, Trans. Amer. Math. Soc. 79 (1955), 256-280. doi.org/10.1090/S0002-9947-1955-0069386-1

[5] J.B. CoOper, "Saks Spaces and Applications to Functional Analysis", Second edition, North-Holland, Amsterdam, 1987. doi.org/10.1016/S0304-0208(08)72315-6

[6] F. Delbaen, J. Orihuela, Mackey constraints for James's compactness theorem and risk measures, J. Math. Anal. Appl. 485(1) (2020), Article 123764 . doi.org/10.1016/j.jmaa.2019.123764

[7] F. Delbaen, K. Owari, Convex functions on dual Orlicz spaces, Positivity 23 (5) (2019), 1051-1064. doi.org/10.1007/s11117-019-00651-x

[8] J. GIL DE LAmadRID, Topology of mappings and differentiation processes, Illinois J. Math. 3 (1959), 408-420. doi.org/10.1215/ijm/1255455262

[9] J. Gómez GIL, On local convexity of bounded weak topologies on Banach spaces, Pacific J. Math. 110(1) (1984), 71-76. doi.org/10.2140/pjm.1984.110.71 
[10] M. GonzÁLez, J.M. GutiÉrRez, The compact weak topology on a Banach space, Proc. Roy. Soc. Edinburgh Sect. A 120 (3-4) (1992), 367-379. doi.org/10.1017/s0308210500032194

[11] A. Horsley, A.J. Wrobel, Efficiency rents of storage plants in peak-load pricing, II: hydroelectricity, LSE, 1999, STICERD DP TE/99/372. http: //sticerd.1se.ac.uk/dps/te/te372.pdf Shortened journal version is at doi.org/10.1016/j.jedc.2006.03.004

[12] A. Horsley, A.J. Wrobel, Localisation of continuity to bounded sets for nonmetrisable vector topologies and its applications to economic equilibrium theory, Indaq. Math. (New Ser.) 11 (1) (2000), 53-61. doi.org/10.1016/S0019-3577(00)88573-0

[13] A. Horsley, A.J. Wrobel, Boiteux's solution to the shifting-peak problem and the equilibrium price density in continuous time, Econom. Theory 20 (3) (2002), $503-537$. doi.org/10.1007/s001990100226

[14] A. Horsley, A.J. Wrobel, Demand continuity and equilibrium in Banach commodity spaces, in "Game Theory and Mathematical Economics", Banach Center Publications, 71, Polish Acad. Sci. Inst. Math., Warsaw, 2006, $163-$ 183. doi.org/10.4064/bc71-0-13

[15] A. Horsley, A.J. Wrobel, "The Short-Run Approach to Long-Run Equilibrium in Competitive Markets: A General Theory with Application to Peak-Load Pricing with Storage", Lect. Notes Econ. Math. Sys. 684, Springer, Berlin-Heidelberg-New York, 2016. doi.org/10.1007/978-3-319-33398-4

[16] M. NowAK, On the finest Lebesgue topology on the space of essentially bounded measurable functions, Pac. J. Math. 140 (1) (1989), 151-161. doi.org/10.2140/pjm.1989.140.155

[17] H.H. SchaEfER, "Topological Vector Spaces", Second edition, SpringerVerlag, New York, 1999. doi.org/10.1007/978-1-4612-1468-7

[18] G. SchlüChtermann, R.F. WheEler, On strongly WCG Banach spaces, Math. Z. 199(3) (1988), 387-398. doi.org/10.1007/bf01159786

[19] A. Wiweger, Linear spaces with mixed topology, Studia Math. 20 (1961), $47-68$. doi.org/10.4064/sm-20-1-47-68

[20] A.J. Wrobel, Bounded topologies on Banach spaces and some of their uses in economic theory: a review, (2020). arXiv:2005.05202 